\title{
Deutsche Biedermeiermedizin, Epoche zwischen Romantik und Naturalismus (1830-1850)
}

\author{
Von K.E. Rотнschuн, Münster (Westfalen)
}

\section{Einleitung und Fragestellung}

Es ist in der deutschen Kulturgeschichtsschreibung gebräuchlich, die Zeit seit dem Ende der Freiheitskriege bis zum Vormärz, also etwa von 1815 bis 1848, als «Biedermeier » zu bezeichnen ${ }^{1}$. Doch ist diese Zeit kulturell durchaus nicht so einheitlich, als daß diese Zusammenfassung zweckmäßig wäre. Dieser Eindruck verstärkt sich, wenn man versucht, auch die Medizin den Wandlungen der kulturgeschichtlichen Strömungen zuzuordnen. Die Zeit zwischen 1815 und 1830 steht noch stark unter den Nachwirkungen der künstlerischen Romantik und der idealistischen Philosophie, aber die Ernüchterung ist deutlich. Gegenständliches Interesse und Neigung zur Empirie verstärken sich. In den Jahrzehnten von 1830 bis 1850 gelangt dieser Zeitgeist der Nüchternheit, Biederkeit, Alltäglichkeit zu seiner Höhe. Nach 1850 setzen sich in Literatur, bildender Kunst und Technik die realistischen Tendenzen durch. Es schien lohnenswert, der Frage nachzugehen, ob diese geistigen Strömungen und ihre Übergänge auch in der Medizin ihren Niederschlag gefunden haben, zumal ob auch in der Medizin zwischen 1830 und 1850 ein «Biedermeier» und um 1850 eine deutliche Wandlung zum Realismus bzw. Naturalismus erkennbar ist. Um diese Frage zu beantworten, bedarf es zunächst eines kurzen Exkurses in die politische und kulturelle Situation jener Jahre.

\section{Die politische Situation}

Wiederherstellung von Frieden und Ruhe, Legitimität und Autorität, Thron und Altar sind die Ziele jener Zeit nach den Freiheitskriegen, in der die Restauration auf allen Gebieten mit unbestrittenem Erfolg durchgeführt wurde. Man knüpft überall an vorrevolutionären Verhältnissen, Lösungen

1 Vgl. dazu Georg Hermann, Das Biedermeier im Spiegel seiner Zeit, Berlin 1931. - Max von Boenn, Biedermeier-Deutschland von 1815-1847, Berlin 1911. - Wilhelm Treue, Illustrierte Kulturgeschichte des Alltags, München 1952. - Franz Schnabed, Deutsche Geschichte im 19. Jahrhundert, 6 Bände, Herder-Bücherei, Frankfurt am Main 1964. 
und Ideen an und hält die politischen, geistigen und kulturellen Errungenschaften der Revolution von den Untertanen fern. Die obrigkeitliche Bevormundung ließ in Deutschland für politische Initiativen des Bürgertums wenig Raum. Die Pressezensur sorgte mit polizeilichen Mitteln für die Unterdrückung der freien Meinungsäußerung. Die wirtschaftlichen Kräfte des Bürgertums waren anfangs noch zu wenig entwickelt, um dem Stand auch politisches Gewicht zu geben. Das bahnt sich erst um 1835 an und verstärkt sich in der Folgezeit. Die bourbonische Restauration in Frankreich findet $1830 \mathrm{im}$ Juliaufstand ihr Ende. Die französische Bourgeoisie nimmt seitdem ihr Geschick wieder selbst in die Hand. Auch in den deutschen Kleinstaaten gärt es damals überall. Die junge Intelligenz erhebt ihre Stimme, doch wird sie in den Demagogenverfolgungen mundtot gemacht. Politische Resignation im Bürgertum ist die Folge; es sucht seinen Ausweg in der wirtschaftlichen Aktivität nach dem Vorbild Englands. 1834 wird der Zollverein unter preußischer Führung gegründet; das begünstigt Handel und Industrie.Auch für Erziehung und Bildung geschieht relativ viel. Im Februar 1848 brechen in Frankreich, im März 1848 in Deutschland und in Wien neue Unruhen aus, getragen von der Intelligenz und dem Bürgertum auf der einen Seite und dem neu entstehenden Proletariat auf der anderen Seite. Zwar blieb das Jahr 1848 wiederum politisch erfolglos, aber das wirtschaftliche und politische Gewicht und das Selbstbewußtsein des Bürgers nahmen in unübersehbarer Weise ständig zu. Gegen die politischen Vorrechte des Adels hatte das Bürgertum zwei wirksame Waffen, erstens seinen Reichtum - gegründet auf Tätigkeit in Handel, Gewerbe und Industrie - und zweitens sein Wissen, seine Kenntnisse, seine Bildung, seine geistige Überlegenheit in vielen wichtigen Bereichen.

\section{Die kulturelle Situation}

Bis um 1830 war Jean Paul ( $†$ 1825) der Abgott des lesenden Publikums, es fand sich in seinen Werken wieder, in der Bereitschaft zu Rührung, zur Sentimentalität. Um 1830 beginnt der literarische Kampf gegen die Romantik $^{2}$. Das «junge Deutschland» wendet sich gegen ihren mangelnden Wirklichkeitssinn. Ihre Wortführer waren Karl Gutzkow, Heinrich Laube, Theodor Mundt, Gustav Kuhn, Heinrigh Heine u.a.m. Um 1830

${ }^{2}$ Vgl. dazu H. W.EPPelsheimer, Handbuch der Weltiteratur, Frankfurt am Main 1960. Hermann Pongs, Das kleine Lexikon der Weltiteratur, Stuttgart 1958. 
stellen die weit verbreiteten Almanache Minerva, Urania, Penelope, Fortuna und wie sie alle hießen, ihr Erscheinen ein. Arnold Ruge gibt seit 1838 die Halleschen Jahrbücher heraus; man bevorzugt handfestes Wissen und wirklichkeitsgetreue Orientierung. Das Konversationslexikon von BrocKHAUS wurde damals das meist gelesene Buch.1839 kommt dazu Meyers Konversationslexikon. Schon 1827 sprach Alexander von Humboldt in Berlin unter größtem Zulauf in der Singakademie vor einem ganz gemischten Zuhörerkreis aus allen Ständen. Der Drang nach Wissen um neue Erkenntnisse, das Interesse an den Ergebnissen der Wissenschaft wächst von Jahrzehnt zu Jahrzehnt. Zwischen 1830 und 1850 entwickelt sich eine neue Literaturgattung, die populärwissenschaftliche Darstellung historischer oder naturwissenschaftlicher Gegenstände. Die erste Auflage von LiEBIGS chemischen Briefen erscheint 1844, im folgenden Jahr beginnt Нuмвоцdтs Kosmos zu erscheinen. Ähnliche Schriften folgen ${ }^{3}$. Gelehrte, Ärzte, Schriftsteller schließen sich in Tagungen und Versammlungen zusammen. Clubs und Kränzchen entstehen, deren Mitglieder oft in Privathäusern zusammenkommen.

Inzwischen beginnen die Städte, ihren Raum zu sprengen. Um 1830 fängt man an, die Ummauerung der Städte einzuebnen, während man bis dahin noch abends die Stadttore geschlossen hielt. Die altdeutsche Tracht wird unmodern. Die Herrenmode beginnt ihre Farbigkeit zu verlieren. Die Welt wird eintöniger, nüchterner, der Alltag beginnt sich zu wandeln. Die erste Eisenbahnlinie von Bedeutung wird 1839 zwischen Dresden und Leipzig eröffnet. Eisenbahn und romantische Naturseligkeit passen aber nicht mehr recht zusammen. Die bürgerliche Initiative führt zur Gründung kleinerer und größerer Unternehmen. Die Einbeziehung der Dampfkraft in die Produktion macht stetige Fortschritte. 1837 gab es in Preußen erst 421 Dampfmaschinen; 1850 die dreifache Zahl ${ }^{4}$. Die Produktion, etwa von Farben, Soda, Schwefelsäure und Chlor, erfolgt noch auf empirischer Basis. Die naturwissenschaftliche Technologie, die Einbeziehung neuer Erkennt-

${ }^{3}$ Damals erschienen in großer Auflage zu erschwingbaren Preisen naturgeschichtliche Atlanten der Tiere und Pflanzen, z.B. zu Okens allgemeiner Naturgeschichte (1843) oder Dr.G.H. von Schuberts († 1860) Naturgeschichte des Pflanzenreichs nach dem Linnếschen System für Schule und Haus, mit prachtvoll kolorierten Tafeln; ferner ohne Autor: Die Naturgeschichte in getreuen Abbildungen und ausführlicher Beschreibung derselben, Halberstadt/Leipzig 1831-1842.

4 Vgl. Friedrich Lütge, Deutsche Sozial- und Wirtschaftsgeschichte, Berlin/Göttingen/ Heidelberg 1960. - Wilhelm Treue, Wirtschaftsgeschichte der Neuzeit, Stuttgart 1962. 
nisse in die Produktion machen nur langsam Fortschritte. Erst nach 1850 wird der Bergbau liberalisiert, und es entsteht die neue Hochofentechnik bei der Eisenverarbeitung. Die bürgerliche Welt schwankt zwischen 1830 und 1850 noch in der Vorliebe zur Vergangenheit, ihren klassischen Idealen, zum Humanismus und fühlt doch das Nahen einer anderen zukünftigen Lebensform. Der Geist jener Jahre bleibt unentschieden zwischen Romantik und Naturalismus. Es sind Jahre des Übergangs. Man liebt die Häuslichkeit, das Leben des kleinen Kreises der Familie; man ist nüchtern, gegenständlich, konkret und hält sich an das Gegebene. Die Malerei schildert neben historischen Stoffen das beschauliche Idyll der Familie, eine hausbackene Welt. Man malt das kleinbürgerliche Alltagsleben in Gassen und Höfen. Zipfelmütze und Schlafrock sind die unentbehrlichen Kleidungsstücke jener Tage. Eine fast weltflüchtige Altväterlichkeit spricht etwa aus den Bildern eines Ludwig Richter (* 1803) oder Karl Spitzweg (* 1808). Nachtwächter, Bücherwürmer, pfeifenrauchende Studenten, Poeten, Sonderlinge, Schulmeister sind ihre Gegenstände, der Alltag ist darin eingefangen, zumal der Feierabend. Spitzwegs Poet stammt aus dem Jahre 1837, der Liebesbrief von 1838. Die Bezeichnung jener Kunstrichtung zwischen Romantik und Realismus als Biedermeier ist umstritten, aber eigentlich nur wegen des negativen Akzents, der das Biedermeierliche fälschlicherweise mit dem Spießbürgerlichen identifizierte.

Die Literatur zwischen 1830 und 1850 hat noch eine stark romantische Unterströmung. Man neigt noch zur Idealisierung der Menschen, allerdings vorzugsweise von solchen der bürgerlichen Welt. Die ländliche, bäuerliche, kleinbürgerliche Welt bildet den Gegenstand dieser Literatur. Man schildert das Leben in der Familie mit den Figuren der trefflichen Mutter, des weisen Großvaters, man verweilt bewegten Herzens bei den Zeugnissen der Kinderliebe, der Einfalt des Herzens, der schlichten Frömmigkeit. Wenn historische Stoffe gewählt werden, dann schildert man nicht die Welt adeliger Helden, sondern die der bürgerlichen Gegenspieler, d.h. der einfachen Leute. Biedermeier ist also traditionsbewußter Bürgerstil, etwas hausbacken, manchmal idyllisch, aber nicht ohne untergründige Dämonie; Leidenschaften und Gefühl sind gebändigt. Die Schilderung der Menschen, der Natur und der menschlichen Umwelt ist schon realistisch, detailliert, mitunter anekdotisch, naturnahe, wie mit den Augen des Malers gesehen. Deutschsprachige Vertreter dieser, auch als «poetischer Realismus» charakterisierten Übergangsepoche ${ }^{5}$ sind z.B. FrIEdrICH RüCKERT (1788-1866), Jeremias GotThelf (1797-1854), ANNETTE von Droste-HüLShoff (1797 bis 1848), Eduard Mörike (1804-1875), Gustav Freytag (1816-1895), Theodor Storm (1817-1888) und GotTfried Keller (1819-1890). Schließlich rechnet man auch AdalberT STIFTER (1805-1868) meistens hierher. Seine Szenerie ist noch oft von romantischer Erfindung durchsetzt. In der Darstellung ist sie schon realistisch. Ein einfaches, menschlich edles,

5 Vgl. Anm. 2. 
leidenschaftsloses und anspruchsloses Milieu wackerer Menschen, eine sehr moralische sonntägliche Welt bilden den Hintergrund seines Werkes. Seine «Studien» entstehen zwischen 1840 und 1845. Der Sinn für die gute hergebrachte Ordnung steht neben gegenständlichem Dingkult. Die Literaturwissenschaft charakterisiert das Biedermeierschrifttum nach G. WEYDT (1931) folgendermaßen: Es schätzt Moral und Sittlichkeit, Unterordnung und Demut, Schickung in das Unvermeidliche, Resignation, Anspruchslosigkeit, Liebe zum Kleinen, zum reifen Alter; es liebt die kleinen Dinge, man sammelt Pflanzen und Tiere, trägt Steine und Zeugnisse der Geschichte zusammen; liebevolle Dingfrömmigkeit ist stärker als der Drang, den Problemen von Natur und Übernatur auf den Grund zu kommen ${ }^{6}$. Auch LeOpold von Rankes Haltung zum Gegenstand der Geschichte entspricht dem Zeitalter. Selbst die Musikgeschichte kennt jenen Umschwung von dem Pathos der Romantik in die Stimmung der Idylle, die sich etwa in Mendelsohns Liedern ohne Worte ausspricht. Die Filigranarbeit Schumannscher Musikstücke (Kinderszenen) gehört ebenso hierher wie die Programmusik eines BerLıoz, die Weichlichkeit eines SpoHr.

So befindet sich der Geist des Biedermeiers zwischen 1830 und 1850 gewissermaßen im Übergang. Man beobachtet mit Liebe die enge kleine Welt und wagt sich noch nicht ins Freie. Von einer neuen Zeit kann man vorerst nur träumen. Das «Biedermeier» war, wie JoEL ${ }^{6}$ a sagt, gewissermaßen die «Siestastunde» des Bürgertums. Die Gefühle der Sekurität überwiegen, sein gewiß vorhandener Freiheitssinn ist meist noch häuslich, nicht provokativ. Nüchternheit, Andacht zum Kleinen und Schwunglosigkeit bis zum Egoismus beherrschen den Geist jener Zeit. Die Pflege des Objektiven tritt in den Vordergrund. Metaphysische Bedürfnisse sterben ab. Die Zeit von 1830 bis 1850 zeigt schon die Ansätze eines literarischen Realismus, erst nach 1850 setzt der Hochrealismus ein.

Der Philosoph Hegel war 1831 gestorben; seine Wirkung auf die breite Öffentlichkeit ließ um 1830 stark nach. Zwei andere Philosophen haben im Deutschland jener Jahre stark gewirkt, Johann Friedrich Herbart $(† 1841)$ und John Stuart Mill ( $†$ 1873), weniger Auguste Comte und A.Schopenhauer. Herbart vor allem in der Psychologie und Pädagogik, Mill mit seinem «System of inductive logic» (1843) im Sinne einer Neubegründung der erfahrbaren Wirklichkeit in der Naturwissenschaft und Medizin; daneben steht Schopenhauer mit seiner Weltverneinung, seinem Pessimismus. Seine Wirkung auf das Publikum setzte erst mit der zweiten

${ }^{6}$ G. Weydt, Literarisches Biedermeier; und W. BietaK, Vom Wesen des österreichischen Biedermeiers, Dtsch.Vjschr. Lit.Wiss. Geistesgeschichte 9 (1931) 628 und 652. Für die Musik vgl. H. Mersmann, Musikgeschichte in der abendländischen Kultur, Frankfurt 1955. ${ }^{6 a}$ KarL Joes, Wandlungen der Weltanschauung, 2 Bände,Tübingen 1934, besonders II, S. 823. 
Auflage der Welt als Wille und Vorstellung nach 1844 ein. Herbart ist eine Brücke zwischen Idealismus und Realismus. Er sucht hinter der Erscheinung das wahre Sein, deren Seinseinheiten er "Reale» nennt.J.St. MiLL ${ }^{7}$ war der bedeutendste Philosoph des Empirismus, einer Erkenntnislehre zur Rechtfertigung des empirisch Gegebenen, Erfaßbaren. Mill sieht seine besondere Aufgabe in der Entwicklung einer Theorie des wissenschaftlichen Beweises durch ein richtiges Vorgehen bei Beobachtung, Empirie und induktiver logischer Verknüpfung. Er behandelt das Wesen der Kausalforschung, die Rolle der Beobachtung und des Experimentes. Es müssen für die Naturzusammenhänge Korrelationen, empirische Gesetze aufgesucht und die beteiligten Umstände in die bedingenden Ursachen zerlegt werden. Seine Philosophie hat, wie noch zu zeigen ist, sowohl auf J. Liebig wie auf Fr. Oesterlen stark gewirkt. In die Jahre zwischen 1840 und 1850 fallen auch die Hauptschriften des Medizinerphilosophen HermanN LотzE $^{8}$ (1817-1881). Sie formulieren schon sehr klar den Weg in die Zukunft. Man gehe aus von den Einzelkenntnissen und suche dazu die kausale Theorie. Man löse das Ganze der Natur in Bruchstücke auf, deren gesetzliche Zuordnung dann erforscht werden muß. Lotze kämpft gegen die Lebenskraft, gegen die Naturheilkraft, gegen die Reizbarkeit, aber er durchbricht 1852 wieder seinen prinzipiellen Mechanismus, indem er die Einwirkungen der Seele auf den Körper zuläßt. Erst die «Materialisten » der nächsten Generation gehen diesen Weg zu Ende. Diese Philosophie des Materialismus gewinnt erst zwischen 1850 und 1860 großen Einfluß, eingeleitet durch Ludwig Feuerbach ${ }^{9}$, aufgenommen in den Physiologischen Briefen von

7 Das Hauptwerk MrLls, $A$ system of logic, ratiocinative and inductive, being a connected view of the principles of evidence and the methods of scientific investigation, London 1843, 2. Ed.,1846. Der Hauptteil des Werkes erschien erstmals in deutscher Sprache als Die induktive Logik. Eine Darlegung der philosophischen Prinzipien wissenschaftlicher Forschung, insbesondere der Naturforschung, Braunschweig 1849; dritte vollständige deutsche Ausgabe, Braunschweig 1868, 2 Bände, (von J.SchIEL).

8 Zu Hermann Lotze vgl. E.Tubbesing, Hermann Lotze. Wurzeln, Werk und Wirkungen seiner mechanistischen Naturauffassung, Diss. Med., Münster 1964. - Lotzes Einstellung war schon früh sehr klar, z.B. in dem berühmten Einleitungsartikel: «Leben, Lebenskraft» von Wagners Handwörterbuch der Physiologie, Band I., Braunschweig 1843, ebenso in Allgemeine Pathologie und Therapie als mechanische Naturwissenschaften, Leipzig 1842, und Allgemeine Physiologie des körperlichen Lebens, Leipzig 1851. - Ders., Medizinische Psychologie oder Physiologie der Seele, Leipzig 1852.

${ }^{9}$ Ludwig Feuerbach (1804-1872), Anti-Hegelianer, Verfasser von Wesen des Christentums (1841). 
Karl Vogt (1845), fortgebildet durch Jаков Moleschotts Kreislauf des Lebens (1852) und durch Ludwig Büchners Kraft und Stoff (1855). Hier ist der Geist des unschlüssig schwankenden «Biedermeiers» zwischen Romantik und Naturalismus überwunden; ein einseitig naturwissenschaftliches Stoff- und Kraftdenken hat sich durchgesetzt. Aber vor 1850 gewinnt dieser Naturalismus erst langsam an Boden. Die damals entstehenden Fabriken und Maschinenhallen werden noch - gewissermaßen schamhaft durch gotische oder griechische Fassaden verkleidet.

Man tut zwar Neues, aber irgendwie noch mit schlechtem Gewissen.

\section{Die Naturforscher des Biedermeierzeitalters zwischen Tradition und Fortschritt} (1830-1850)

Wenn wir hier zuerst von Alexander von Humboldt (1769-1859) ${ }^{10}$ sprechen, dann nicht deswegen, weil er ein Kind des Biedermeierzeitalters gewesen wäre. Das Gegenteil ist richtig. Humboldt war seit seiner Jugend immer ein Mann der Beobachtungslust, ja der Beobachtungssucht und des Versuchs, und nie ein Gefolgsmann der Naturphilosophie.Aber er war kein Künstler des Experimentierens, ja er war dabei ungewöhnlich ungeschickt (Rothschuh, 1959) ${ }^{11}$. Er wollte «keine rationelle Wissenschaft der Natur»; was er anstrebte, war «die denkende Betrachtung der durch Empirie gegebenen Erscheinungen als eines Naturganzen $" .^{12}$ So heißt es im Kosmos 1845. «Die Erfahrungswissenschaft ist letztes Ziel menschlicher Forschung ${ }^{13}$. Humboldt war das Idol jenes hier behandelten Zeitraumes, ein Mann mit unglaublicher Wissensbildung, der tausende Arten von Pflanzen und Tieren gesammelt, die Meteorologie, die Pflanzengeographie um zahllose Daten bereichert hatte. Sein Kosmos (1845-1858) wurde das Buch der Epoche. Die beschreibende, keineswegs die experimentelle Naturwissenschaft war seine Stärke.

10 Vgl. zu Alexander von Humboldt u. a. Hanno Beck, Alexander von Humboldt, Band 1 1959, Band 2, 1961, Wiesbaden. - Heinrich Schipperges, Humboldts Beitrag zur Medizin des 18. Jahrhunderts, in Alexander-von-Humboldt-Festschrift, herausgegeben von J.H. Schultze, Berlin 1959, S. 36-68.

11 Karl E. Rothschun, Alexander von Humboldt und die Physiologie seiner Zeit, Sudhoffs Arch. 43 (1959) 97-113.

12 Alexander von Humboldt, Kosmos. Entwurf einer physischen Weltbeschreibung, 4. Bände, Stuttgart/Tübingen 1845-1858, besonders Band I, 1845, S. 31.

13 Ebenda, S. 32. 
Unter den deutschen Naturforschern galt Justus LieBig (1803-1873) damals seit seinen bedeutenden Werken von 1840 und $1842^{14}$ als einer der bekanntesten. Sosehr er mit den Verfahren des Chemikers die Bausteine der Körperwelt, der anorganischen und der organischen aufzuklären, ihre Morphologie zu enträtseln und ihr Zusammenwirken zu interpretieren wußte, so konnte er sich doch niemals aus der Widersprüchlichkeit zwischen einer gleichzeitigen Wirksamkeit chemischer Kräfte und einer organisierenden Lebenskraft befreien ${ }^{15}$. Er konnte sich nicht dazu verstehen, auf eine Lebenskraft als Ursache der spezifischen Struktur der Organismen und der Wirkungsart ihrer Substanzen zu verzichten, trotz allen Spotts, mit dem ihm die junge Generation über den Mund fuhr, etwa E. du Bois-Reymond (1848) ${ }^{16}$ oder gar Jakob Moleschott (1863). Dieser interpretierte Liebigs Auffassung so: "In den organischen Körpern muß eine Ursache wirken, welche die gerade Linie krumm biegt ». ${ }^{17}$ Liebig sagt 1865 : «in den Organismen wirkt eine Kraft, welche die stärksten chemischen Anziehungen aufhebt und geradezu umkehrt», ${ }^{18}$ oder «im Organismus der lebendigen Pflanzen verlieren Luft, Wasser, Sauerstoff und Kohlensäure ihren chemischen Charakter und üben weder durch ihre Masse noch durch ihre Affinität eine Wirkung aus ». ${ }^{19}$ Andererseits spottet Liebig in den chemischen Briefen über die Vitalisten, die sich mit dem Worte Lebenskraft einfach alle Erscheinungen erklären, die sie nicht verstehen. Kurz, seine Haltung ist schwankend und widerspruchsvoll ${ }^{20}$. Ganz anders etwa die Physiologen Theodor Schwann (1839) oder E. du Bois-Reymond (1848).Andererseits bekennt sich Liebig

14 Justus Liebig, Die organische Chemie in ihrer Anwendung auf Physiologie und Pathologie, 1. Auflage, Braunschweig 1842 (seit 3. Auflage: Thierchemie). - Ders., Die Chemie in ihrer Anwendung auf Physiologie und Pathologie, Braunschweig 1840.

15 Der 12. Brief in Liebigs Chemische Briefe, 1. Auflage, Heidelberg 1844, über die Rolle der Lebenskraft ist wörtlich identisch mit dem 16. Brief der Volksausgabe zwanzig Jahre später (1865).

16 DU BoIs-ReYmond in der berühmten Vorrede zum Band I seiner Untersuchungen zur thierischen Elektrizität 1848, bes. S. XXXIV-L, oder in der Gedächtnisrede auf Јон. Müller, siehe Anm. 19, S. 208.

17 Јаков MоцеSснотт, Der Kreislauf des Lebens. Physiologische Antworten auf Liebigs Chemische Briefe, 1. Auflage, Mainz 1852, 4. Auflage, Mainz 1863, S. VI.

18 LieBig, Chemische Briefe, Volksausgabe, Leipzig/Heidelberg 1865, S. 203 (23. Brief).

19 Ebenda, S. 200 (23. Brief).

20 Wenn Liebig (1844, S.148) sagt, die Lebenskraft sei «eine Kraft der Bewegung, fähig, ruhenden Atomen eine Bewegung mitzuteilen ...», so bemerkt dazu MoLeschотт 1863, S. 379: «Wer von einer Lebenskraft redet ... ist genöthigt, eine Kraft ohne Stoff anzunehmen. Eine Kraft ohne stofflichen Träger ist eine wesenlose Vorstellung ...» 
(1846) zu Mills Methodologie der wissenschaftlichen Forschung ${ }^{21}$. Er schildert dessen Hauptgedanken über Naturgesetze, induktive Logik, über die möglichen Arten von Fehlschlüssen, über Worterklärungen, aber die Aneignung ging ihm damals augenscheinlich noch nicht bis unter die Haut. E. du Bois-Reymond, seinem Jahrzehnt weit voraus, formuliert 1848 schonungslos seine Kritik an jener alten Lebenskraft, der auch sein Lehrer, Johannes Müller, bis zu seinem Tode anhing ${ }^{22}$. Die neue Generation der um 1815 bis 1821 Geborenen, wie Wunderlich, Ludwig, du Bois-Reymond, Helmholtz, Virchow, Lotze, hatte das alles hinter sich gelassen. Sie bereiten in den vierziger Jahren dem Geist der naturalistischen Epoche nach 1850 den Weg.

Auch in seinem Streben nach Universalität, mit der Tendenz, eine Riesenmasse von Erfahrungsmaterialien zusammenzutragen und trotzdem «das Ganze der Lebenserscheinungen mit hochschwebenden Blick zu beherrschen » ${ }^{23}$, war Müller ganz ein Kind dieses Übergangszeitalters, in seinen Tendenzen denen von Alexander von Humboldt sehr ähnlich.

Nicht ohne Grund sei unter den hervorragenden Naturforschern der Biedermeierzeit die Gestalt von Johannes Müller (1801-1858) etwas näher untersucht. Johannes Müller war ein leidenschaftlicher, genialer Beobachter und Zergliederer, weniger ein Experimentator ${ }^{24}$. Er traute dem eingreifenden physiologischen Experiment nicht recht. Das hing damit zusammen, daß er die Veränderungen an tierischen Körpern unter äußeren künstlichen Bedingungen nicht als « Wirkungen », sondern als « Reaktionen », bestimmt vom Organismus, ansah. In Müllers Grundri $\beta$ der Vorlesungen über die Physiologie (Bonn 1827) heißt es: «Da alle äußere Einwirkung auf den lebenden Organismus nicht eine Wirkung desselben hervorbringt, in welcher die äußere Bedingung enthalten wäre, da vielmehr jede Lebensäußerung eine durch die äußere Ursache nur bedingte, dem Organismus selbst eigentümliche Energie desselben ist, so ist der Schluß von einer künst-

${ }^{21}$ In Die Thierchemie oder die organische Chemie in ihrer Anwendung auf Physiologie und Pathologie (3. Auflage der Die Organische Chemie ...), Braunschweig 1846. Auf S. 141-231 geht LIEBIG unter dem Titel «Die Methode » ausführlich auf Mills Erfahrungsphilosophie ein.

${ }^{22}$ Vgl. E. DU Bors-ReYmond, Gedächtnisrede auf Johannes Müller (1858), in Reden, Band I; 2. Auflage, Leipzig 1912, S. 205.

${ }^{23}$ So drückt es du Bors-ReYmond, ebenda, S. 266 aus.

${ }^{24} \mathrm{Zu}$ JoH. Müller vgl. K. E. Rothschuн, Geschichte der Physiologie, Göttingen/Berlin/ Heidelberg 1953, bes. S. 112 ff., ferner ders., Johannes Müller und Carl Ludwig, Dtsch. Med.Wschr. 1953, S.71-74. 
lich gesetzten Bedingung auf eine dadurch bewirkte Lebensäußerung höchst mißlich, insofern nicht diese letztere wieder rein beobachtet wird»(V). Müller kommt eben noch aus der naturphilosophischen Denkweise.Aber er ringt sich durch zu einer Beobachtungsphysiologie und -morphologie. Im Jahre 1830 ergreift er noch einmal das Wort zur Methodologie und fordert, daß man « unermüdet sei im Beobachten und Erfahren ». ${ }^{25}$ «Beständen alle unsere Erfahrungen », so meinte Müller damals, «aus solchen Beobachtungen, so wäre alles weitere Theorisieren unnötig, und die Theorie wäre eine schlichte Erzählung der Tatsachen, von denen eine die Konsequenz der anderen ist.» Eine weitere Hoffnung ließ Müllers Konzept von der spezifischen Energie auch gar nicht aufkommen. Im Banne der Tradition, die über Haller, Brown, Schelling, v. Walther zu ihm hinführt, ist jede Einwirkung ein Reiz, der immer zu einer spezifischen, also durch die lebendige Organisation bestimmten Antwort führen muß. Dadurch wird der Reiz seiner naturwissenschaftlichen Dimension als Ursache (mechanisch, thermisch, akustisch, stofflich) entkleidet und seine Wirkung, die Reaktion, wird ihres eindeutigen Charakters beraubt.

Weil das so war, lehnten Wunderlich und andere das Reiz-Reaktionskonzept als Basis der naturwissenschaftlichen Medizin ab.Auch Henle fordert eine Kritik des Begriffs der «Reaktion» (1844) ${ }^{26}$.

Noch in seinem Handbuch der Physiologie ${ }^{27}$ hat Müller diesen Standpunkt genau expliziert. Seine Theorie des Organismus ist konsequent aufgebaut auf der Idee der Lebenskraft und der Reiz-Reaktionsdynamik. Die Lebenskraft, eine vis essentialis, äußert sich als Vegetationskraft, Empfindungskraft, Bewegungskraft, Nervenkraft ${ }^{28}$. Sie beseelt den Keim. Sie entfaltet eine «bewußtlos wirkende (aber gesetzliche) zweckmäßige Tätigkeit». Organische Stoffe entstehen nur dank der Mitwirkung dieser eigentümlichen Kraft. Sie bestimmt die Stärke der Organtätigkeit auf einen Reiz. Selbst «Nahrungsmittel ... sind Reize, welche beleben und selbst belebt werden können ».29 Beim Atmen gehen Lebensreize in das Blut über ${ }^{30}$. Die Reaktion

${ }^{25}$ In Bildungsgeschichte der Genitalien, 1830, darauf weist DU Bors-REYMond in seiner Gedächtnisrede (vgl. Anm. 22), S.162, besonders hin.

26 Aber Henle kam von dem Reiz-Reaktions-Gedanken auch nicht ganz los, wie ihm LIEBIG 1846 (vgl. Anm. 21), S. 154-160, vorwarf.

27 Johannes MüLler, Handbuch der Physiologie des Menschen, 1. Band, Coblenz 1833/34, 825 Seiten, 2. Band 1837/38/40 in mehreren Teilen.

${ }^{28}$ Ebenda, Band 1, S. 9.

${ }^{29}$ Ebenda, Band 1, S. 31.

30 Ebenda, Band 1, S. 32. 
ist von der Natur des Reizes ganz verschieden, sie ist Äußerung einer Lebenseigenschaft eines Organes ${ }^{31}$. «Die Physiologie gewinnt ebenso sichere Empirie wie die übrigen Naturwissenschaften, wenn sie die eigenthümliche Reaktionsart aller Theile des thierischen Körpers kennt $\gg{ }^{32}$ Es ist klar, daß eine solche Einstellung keinen kausalgesetzlichen Determinismus erlaubt, sondern höchstens eine empirische Regelmäßigkeit erwarten läßt.

Dagegen nahmen C.A. Wunderlich (1842) und Friedrich Oesterlen ${ }^{33}$ (1843) Stellung. Gewiß setzte die Lehre von der Reizbarkeit positive Untersuchungen anstelle eines Kräftevitalismus, aber sie wurde mit der Zeit zur Worterklärung. Die Reaktion war gewissermaßen eine objektivierte Gegenhandlung der lebendigen Organisation, man sprach vom Willen als Reiz der Nervenerregung, der Entzündung als eines sensitiven Nervenreizes, der Luft als Reiz für die Respirationsorgane. Kein Organ funktioniere, ohne daß es gereizt wird und eine zwecktätige, nicht nur zweckmäßige Antwort abgibt. Der Stoffwechselprozeß ist, wie Friedrich Oesterlen (1812-1877) damals in einer interessanten Jugendschrift (1843) ausführte, kein Reizvorgang ${ }^{34}$. Funktionen entstehen nicht durch Reize, sondern werden durch sie modifiziert. Müllers Konzept gehört also noch der mehr empirischen, voranalytischen und teleologischen Denkweise der Physiologie an. Er gehört in das Zwischenreich des Biedermeiers, auch mit seiner Vorliebe für die vergleichende Anatomie und Physiologie, die ihre Generalisierungen manchmal glücklich, manchmal fehlerhaft durch Analogieschlüsse und nicht durch Kausalanalyse gewinnt. Die nächste Generation sieht in der Lebenskraft, der Nervenkraft, der Reaktionskraft, der seelischen Kraft verbale Erläuterungen, keine zureichenden Erklärungen $\mathrm{mehr}^{35}$.

31 Ebenda, Band 1, S. 54.

32 Ebenda, Band 1, S. 35.

${ }^{33}$ Vgl. dazu K. E. Rотнsснuн, Friedrich Oesterlen (1812-1877) und die Methodologie der Medizin, Sudhoffs Arch. 1968, im Druck, mit Biographie und Bibliographie.

34 Siehe Oesterlen, Zur Lehre von der sogenannten Reizbarkeit und Reizung - als Beitrag zu einer positiven Pathogenese, Beiträge zur Physiologie des gesunden und kranken Organismus, Jena 1843, S. 101-160. Der Organismus ist nicht von außen bestimmt, sondern er besitzt auch Eigentätigkeit (119). Die vitale Reizbarkeit ist eine Sache der Organisation. Die Funktion ist Folge der nutritiven Vorgänge (125).

35 Diese Stellungnahme zur Lebenskraft findet man zwischen 1840 und 1850 immer häufiger, z. B. in C.A. Wunderlichs Handbuch der Pathologie und Therapie, Band 1, Stuttgart 1850, S. 6; bei Fr. Oesterlen, Med. Logik, Tübingen 1852, p. 458. 


\section{Die klinische Medizin des Biedermeierzeitalters}

Auch die praktische Medizin jener Jahre zwischen 1830 und 1850 läßt die Kennzeichen des Übergangs zwischen zwei Zeitaltern erkennen. Die spekulative Medizin ist weitgehend überwunden, alles drängt zurück zur nüchternen Beobachtung am Krankenbett, zur Empirie. Die vielen neuartigen klinischen Systeme hatten letztlich alle enttäuscht und in der Krankheitslehre eine große Unsicherheit hinterlassen. Der praktische Arzt hatte jeden zuverlässigen Ratgeber auf seinem komplizierten Gebiet und jeden festen Boden unter den Füßen verloren. Eine allgemeine Skepsis breitete sich aus, zunächst gegenüber der alten Krankheitstheorie, dann auch gegen die altehrwürdigen, übernommenen Formen der Therapie, besonders der Materia Medica. Pierre Louis in Frankreich hatte die numerische Methode am Krankenbett eingeführt und u. a. gezeigt, daß der Aderlaß sein Renommee zu Unrecht besitzt ${ }^{36}$. Die neuere pathologische Anatomie zeigte die Existenz schwerer, kaum reparabler Veränderungen an den inneren Organen bei Tuberkulose (Lænnec), bei Nierenleiden (Bright), bei Leberkrankheiten (Rokitansky), bei Herzleiden (Bouillaud) usw. Diese Tatsachen verstärkten die Entmutigung der Ärzte, sie zerstörten das Vertrauen in ihre Kunst. Von allgemeiner Skepsis bis zum therapeutischen Nihilismus war es nicht mehr weit. Das ist für die Medizin in Paris (Ackerknecht) ${ }^{37}$ und Wien (E. Lesky) ${ }^{38}$ wohlbekannt. Aber in Deutschland war es auch nicht viel besser ${ }^{39}$. In der allgemeinen Lähmung fanden alle jene willige Ohren, die zur Restauration der hippokratischen Medizin aufriefen, zur Erfahrungsheilkunst, zur empirischen Medizin. Diese empirische Medizin ist für Deutschland das Pendant zum Zeitgeist des Biedermeiers. Sie ist die

36 Pierre Charles Alexandre Louis (1787-1872), Recherches anatomiques ... de GastroEntérite,T.II, Paris 1829, IV e Partie,Traitement. Der Aderlaß ist nach numerischer Methode ohne Einfluß auf den «cours de la maladie», S.459-476; vgl. zu Louis auch ED. Rud. Müllener, Pierre Charles Alexandre Louis (1787-1872). Genfer Schüler und die «méthode numérique», Gesnerus 24 (1967) 46-74.

37 E.H.Ackerknecht, La médecine à Paris entre 1800 et 1850, Conf. du Palais de la Découverte, Nr. 58, Paris 1958. - Ders., Die Therapie der Pariser Kliniken zwischen 1795 und 1840, Gesnerus 15 (1958) 151-163.

38 E.LESKy, Die Wiener Medizinische Schule im 19.Jahrhundert, Graz/Köln 1965.

${ }^{39}$ Die Entwicklung der therapeutischen Skepsis in Paris, Wien und Deutschland hat Julius Petersen in Hauptmomente in der geschichtlichen Entwicklung der medizinischen Therapie, Kopenhagen 1877, sehr detailliert geschildert; auch Wunderlichs Weg vom Rationalismus des Jahres 1842 zur kritischen Empirie des Handbuches 1850. 
Hoffnung nicht nur der älteren Ärzte, sondern spielt auch noch im Denken der jungen Revolutionäre der deutschen Heilkunde, etwa bei Carl August Wunderlich, bei Jakob Henle anfangs eine nicht zu übersehende Rolle. Die Schwächen der Empirie sind der Ausgangspunkt ihres Umdenkens. Die Tatsache, daß das Enchiridion Medicum ${ }^{40}$ von Chr. W. Hufeland von 1836 bis 1857 zehn Auflagen erlebte, bezeugt die große Zustimmung der praktizierenden Ärzte zu diesem Werk der «Erfahrungsheilkunde». Seine Krankheitslehre fußte auf dem Reiz-Reaktions-Denken und ist nahe verwandt der Denkweise von Johannes Müller. Die Krankheit entsteht durch «erregende» Einwirkungen, die je nach dem Genius epidemicus oder Ort variieren, aber ihr Bild ist bestimmt durch die besondere Reaktionsweise der nach 12 Konstitutionen klassifizierbaren Kranken. Nicht so sehr die Ursachen der Krankheit als die Antwort der Kranken bestimmen Krankheitsart, -schwere, -verlauf, -heilung. Natürlich war Hufeland (1762-1836) kein Arzt des Biedermeiers, aber sein Denken entsprach ganz den Bedürfnissen des Zeitalters ${ }^{41}$.

Empirisch war in den ersten Jahrzehnten des 19. Jahrhunderts auch die französische Medizin (Ackerknecht) ${ }^{42}$. Die Pariser Beobachtungs- und Krankenhausmedizin suchte nach Beobachtungszusammenhängen, nicht nach Kausalzusammenhängen. Die großen Franzosen waren meistens aus der Schule von Cabanis hervorgegangen und verschmähten es, nach «primären Ursachen » zu forschen. Pinel und Bichat haben eine Aversion gegen viel Theorie und Raisonnement. Auch Lænnec gehörte zu diesen ausgesprochenen Empirikern. Ihr Ziel ist der Ausbau der Korrelationen zwischen Symptomen und organischer Veränderung, die Beschreibung der Phänomene und nicht die Analyse der verborgenen Prozesse. Sie schätzen weder das

40 Chr. W. Hufeland, Enchiridion Medicum, Berlin 1836.

41 Hufeland hat unter dem Einfluß von Haller und Brown in seiner Schrift Ideen über Pathogenie und Einflu $\beta$ der Lebenskraft auf Entstehung und Form der Krankheiten (Jena 1795) schon im Kern ähnliche Vorstellungen entwickelt wie 1836. «Es gibt also keine pur mechanische oder chemische Wirkungsart auf oder in dem belebten Körper, sondern alles ist animalistisch, d.h. es trägt den Karakter des Lebens» (23). Ein Reiz ist etwas, was die Lebenskraft und ihre Rezeptivität affiziert (20). «So verschieden und mannigfaltig auch die Krankheitsursachen und Reize seyn können, so ist die Krankheit selbst doch immer das Produkt der durch jene Reize erst erregten und auf gewisse Art geäußerten Lebenskraft» (47). "Die Reaktionsweise ist der Hauptpunkt der Pathogenie» (151).

42 E.H.Ackerknecht, Medicine at the Paris Hospital,1794-1848, Baltimore 1967. - In Paris wurde 1832 die «Société Médicale d'Observation» gegründet. 
Mikroskop noch die Chemie noch das physiologische Experimentieren. Und wenn sie es tun, dann zur Bereicherung der beobachtbaren Zusammenhänge unter den Lebenserscheinungen, nicht zur Analyse der kausalen Betriebsstücke.Auch hier herrscht mehr Zurückhaltung und Skepsis als mutiges Voranschreiten ins Unbekannte. In dieser empirischen Medizin marschierten, wie Claude Bernard einmal sagte (1865) ${ }^{43}$, Physiologie, Pathologie und Therapie getrennt nebeneinander her. Erst durch das Experiment sollten die Wege zwischen ihnen gebahnt werden, das wurde aber erst in der zweiten Jahrhunderthälfte allen deutlich.

Zu der Generation der Ärzte zwischen Romantik und Naturalismus gehört auch JohanN Lukas Schönlein (1793-1864). Seine naturhistorische Theorie der Krankheiten gehört noch ganz in die naturphilosophische Richtung, aber seine Pathologie und Therapie (1837) ${ }^{44}$ ist beste empirische Medizin. Er benutzt alle Hilfsmittel zur Erweiterung der Krankenbeobachtung wie Inspektion, Palpation, Auskultation, Perkussion, Pupillenweite, Blutfarbe und -beschaffenheit, Harnfarbe und -menge sowie Sediment, Sputum, Pulszählung, Sprache, Gang, Haltung, Sinnesorgane, aber alles ohne messendes Verfahren, ohne Thermometer, ohne Waage, ohne Harnchemie, ohne Blutuntersuchungsverfahren, ohne Hilfsmittel der Naturwissenschaften, alles deskriptiv. Die Symptome stehen unverbunden neben dem pathologischen Befund, dem Verlauf und der Therapie. Das ist blendende Empirie, aber noch keine naturwissenschaftliche Betrachtungsart im Sinne der zweiten Jahrhunderthälfte. Das ist echte Biedermeiermedizin. Gewiß ist jenes Zeitalter der Biedermeiermedizin zwar außerordentlich beeindruckt von der Zuverlässigkeit naturwissenschaftlicher Aussagen auf physikalischem und chemischem Gebiet. Man hofft auch auf gleich zuverlässige Resultate einer naturwissenschaftlichen Medizin. Man ahnt auch die Morgenröte einer neuen Zeit und sucht, ihr entgegenzugehen, aber weiß noch nicht den Weg. Man weiß, daß es nur über das exakte Experiment gehen kann, aber wie soll man am Menschen oder auch nur am Tier mit seiner Fülle unbekannter Zusammenhänge mit verwertbaren gesetzlichen Resultaten experimentieren? Selbst die Bahnbrecher der Zukunft bleiben hier nur in gewissen Postulaten stecken. C.A. WunderLich, seit 1842 ein

${ }^{43}$ In Introduction in deutscher Übersetzung Einführung in die experimentelle Medizin (Sudhoffs Klassiker der Medizin, Band 35, Ed. K. E. RoтнsснUн), Leipzig 1961, S. 16.

44 Johann Lukas Schönleins allgemeine und spezielle Pathologie und Therapie. Nach den Vorlesungen, 4 Bände, 3. Auflage, Herisau 1837 (1. Auflage 1832). 
krasser Gegner jeder Empirie ${ }^{45}$, rügt 1850 die «unvorteilhafte Hast» der Anwendung mechanischer und chemischer Prinzipien für den Organismus $^{46} .1851$ empfiehlt er sogar die 1842 verurteilte Massenbeobachtung und Statistik ${ }^{47}$. Klarer sind J. Henles Vorschläge für die Zukunft einer rationellen Medizin ${ }^{48}$ (1847), die «die Absicht hat, sich sowohl von den Ursachen der Phänomene als der Wirkungsweise der Mittel Rechenschaft zu geben». Das Experiment setzt willkürlich die Ursache, beobachtet die Folgen und sucht die richtige Beziehung dazu. Man muß auf analytischem Wege zu dem einfachen Grund der Krankheiten vordringen, dann den Ursachen entgegenwirken ${ }^{49}$. Das ist aber alles sehr theoretisch. Jakob Henle überschreibt seinen Einleitungsaufsatz im Band 1 (1844) seiner Zeitschrift für rationelle Medizin mit «Medizinische Wissenschaft und Empirie». «Der Arzt ermittele empirisch das 'Zusammenvorkommen' und versuche dann, zur Erkenntnis des 'inneren Zusammenhanges' fortzuschreiten. Er muß also empirisch und rationell verfahren.» ${ }^{50}$ Auch VIRchow hält im Jahre 1847 die Empirie noch für die unvermeidbare Haltung des praktischen Arztes: «Wir kennen die Bedingungen, unter welchen ganze abweichende Erscheinungsreihen in den lebenden Körpern auftreten, noch ganz unvollkommen ... Unter diesen Verhältnissen hat der praktische Arzt das Recht, einem gewissen Empirismus zu huldigen, aber er hat noch viel mehr die Verpflichtung, durch eigene Beobachtung diesen Empirismus vernichten und den glorreichen Bau der wissenschaftlichen Medizin aufführen zu helfen. ${ }^{51}$ Einige Praktiker gründeten 1847, angeregt von Johann Gottfried Rademacher, dem Propheten der empirischen Medizin, eine eigene Zeitschrift für Erfahrungsheilkunst, um durch Sammeln von Beobachtungen über viele nach Symptomen und Therapie vergleichbare Fälle mehr Sicherheit in die Therapie zu bringen. «Die empirische Methode der Forschung mit ihrem mächtigsten Hebel, dem Experiment, ist allein geeignet, die Vervoll-

45 Carl August Wunderlich, Einleitung des 1. Bandes von Archiv für physiologische Heilkunde, von W. Roser und C.A. WuNDERLICH, 1842, S. I-XXX.

46 Ders., Handbuch 1, siehe Anm. 35.

47 Ders., Ein Plan zur festeren Begründung der therapeutischen Erfahrungen. Antrittsvorlesung, gehalten zu Leipzig den 12. März 1851.

48 Јаков Henle, Medizinische Wissenschaft und Empirie, Z. rationelle Medizin 1 (1844) 1-35, bes. S. 23.

49 Ebenda, S. 26.

50 Ebenda, S. 35.

51 Rudolf Virchow, Über die Standpunkte in der wissenschaftlichen Medizin, Arch.pathol. Anat. Physiol. klin. Med.1 (1847) 3-19. 
kommnung der heilenden Kunst wahrhaft zu fördern » ${ }^{52}$, aber unter diesem Experiment ist die klinische Beobachtung am Krankenbette, der therapeutische Vergleichsversuch gemeint, der die Kriterien eines naturwissenschaftlichen Experiments in keiner Weise erfüllt. Fr. Löffler, einer der Herausgeber, empfiehlt, ein- und dasselbe Arzneimittel an den verschiedensten Krankheitsformen auszuprobieren ${ }^{53}$. Gesucht werden die erfahrungswissenschaftlichen Gesetze der Krankheitslehre und der Heillehre in Übereinstimmung mit physiologischen Gesetzen.

Von ganz besonderem Interesse ist der Versuch von Friedrich OesterLEN, in seiner Medizinischen Logik (1852) eine Methodologie der Medizin auf der Basis der neuen induktiven Erfahrungswissenschaft im Gefolge von J.St.Mill zu entwickeln. Die Methode setzt sich aus zwei Akten zusammen, der Beobachtung und der Generalisation. Die Beobachtung führt zur Feststellung der Coexistenz von Erscheinungen mit statistischer Sicherung. Die Analyse, die Zerlegung der Erscheinungen in die elementaren Einzelfaktoren, ist in der Medizin praktisch nicht ausführbar, bestenfalls gedanklich ${ }^{54}$. Das Experiment unter künstlichen Bedingungen wird in der Medizin selten und mangelhaft angewandt. Man muß glücklich sein, wenn man zu «empirischen Gesetzen» gelangt ${ }^{55}$. Dazu verhelfen Statistik und numerische Methode ${ }^{56}$.

Dieser Medizin ist es also trotz aller Ahnung, daß nur ein naturwissenschaftliches Kausaldenken geeignet ist, die Unsicherheiten der Medizin zu überwinden, noch ganz unklar, wie man das machen soll. Die Empirie bleibt vorerst beherrschend. Die Biedermeiermedizin setzt ihre Hoffnung also zunächst noch auf eine geordnete Sammlung von Erfahrungsmaterialien; sie ist skeptisch, nüchtern und noch unsicher gegenüber den Möglichkeiten des kausalanalytischen Experiments. Sie bleibt vorexperimentell, sosehr sie auch jene Zuverlässigkeit experimentell gesicherter Zusammenhänge, zumal für die Therapie, als Hoffnung für die Zukunft artikuliert. Einige Ärzte sehen in diesem Dilemma einen zweiten, greifbareren Ausweg. $\mathrm{Da}_{n}$ die Arzneimitteltherapie in ihrer ganzen Unsicherheit wenig Hoffnung

52 Zeitschrift für Erfahrungsheilkunst, herausgegeben von Dr. A. BernHardi und Dr. Fr. LöFFLER, 1 Band (1847/48), S. 5.

53 Fr. Löffler, Die Medizin als Wissenschaft und die Bedingungen ihres Fortschritts. Ein Beitrag zur ärztlichen Grundsatzfrage, Erfahrungsheilkunst 1 (1847/48) 1-111, bes. 31.

54 Fr. Oesterlen, Medizinische Logik, Tübingen 1852.

55 Ebenda, S. 141-174, bes. S. 164.

56 Ebenda, S. 129 ff. 
bietet, wird die Medizin ganz andere Wege zu beschreiten haben. Hier ist es in Deutschland wieder Fr. Oesterlen, der einen neuen Weg zeigt und beschreitet. "Statt hunderterlei Leiden und Seuchen so oft vergeblich zu bekämpfen, müßten wir sie vielmehr lieber verhüten oder durch eine gesundheitsgemäße Stellung aller Lebensverhältnisse, nicht gerade bloß durch Arzneien und dergleichen zu beseitigen lernen", heißt es in Oesterlens Handbuch der Hygieine (1851/V), aber wenig später: «anstelle des Therapeuten vom alten Schlag muß wohl mehr und mehr der Prophylaktiker treten» (1852) $)^{57}$. Konsequenterweise geht Oesterlen jetzt ganz auf das Gebiet der Hygiene oder Vorbeugung über. Sein Handbuch der Hygieine (1851) bringt in Anlehnung an französische Vorbilder, insbesondere M. LÉvY $(1845)^{58}$, eine Schilderung der verheerenden Wirkung der schlechten Lebensbedingungen der Arbeiterklasse mit allen ihren Folgen für Krankheitshäufigkeit und Sterblichkeit. Wie Lévy gliedert er die Hygiene in einen privaten und einen öffentlichen Anteil. Wie Lévy vertraut Oesterlen nur auf die Statistik und auf die Auswirkung der öffentlichen Hygiene. Lévy meint «elle constitue, à vrai dire, la seule médecine possible parmi les masses.» ${ }^{59}$ Während aber Lévy den Stoff rein deskriptiv behandelt, trägt Oesterlen mit Leidenschaft die Forderung vor, durch private und öffentliche Maßnahmen die Lebensbedingungen der Arbeiterklasse zu verbessern und damit die Voraussetzungen zur Erhaltung und Wiederherstellung der Gesundheit zu schaffen. "Hilft es doch zu Nichts, schlechthin Gesundheitsregeln zu geben; es muß auch allen Volksklassen so gut als dem Einzelnen die Möglichkeit gegeben sein, unseren unabweislichen Lebens- und Gesundheitsbedingungen oder mit anderen Worten den Gesetzen unserer Natur Genüge zu thun. ${ }^{60}$ Seine Hygieinischen Briefe (1859) fordern noch einmal anstelle der alten Medizin die Maßnahmen, die Menschen und Völker gesund zu erhalten ${ }^{61}$. Sein Handbuch der medizinischen Statistik 1865 bekräftigt das Bekenntnis zur empirischen Methode der Statistik: «Sollte sich die Krankheitslehre je zum Rang einer exakteren Wissenschaft, die Medizin zu einer

57 Ebenda, S. 519.

58 Michel LÉvy, Traité de l'hygiène publique et privée, 1.Auflage, 2 Vol., 1845.

59 Ebenda, T. I, p. 52.

60 Friedrich Oesterlen, Handbuch der Hygieine für den Einzelnen und für die Bevölkerung, Tübingen 1851, S.V. Die Leistungen Oesterlens werden von Jul. Petersen (vgl. Anm. 39) ganz besonders hervorgehoben. Die Hygiene ist nach OEsterLen eine «politische Wissenschaft».

61 Fr. Oesterlen, Der Mensch und seine physische Erhaltung. Hygieinische Briefe für weitere Leserkreise, Leipzig 1859, Vorwort. 
verhältnismäßig sicheren Kunst erheben können, so würden sie zweifelsohne vor allem nur der Statistik zu danken haben.» ${ }^{62}$ "Und hierin gerade liegt die wahre unendliche Bedeutung der Statistik. Gibt sie uns auch noch kein sicheres Verständnis, keine Wissenschaft, so ist sie doch in einem Gebiet wie das unsere der einzig mögliche Weg dazu, indem sie allein das hierzu erforderliche Erfahrungsmaterial zu liefern vermag. ${ }^{63}$ Mit der Forschung nach besseren Lebensbedingungen für das Proletariat berührt sich Oesterlen mit jener Ärztegeneration um die Mitte des 19. Jahrhunderts, die wie Virchow (1848) eine Reform der mangelhaften Gesellschaft ebenso wie eine Reform der ärztlichen Versorgung der arbeitenden Bevölkerung fordern. Seuchen sind keine Sendung der Götter, sondern Mängel der gesellschaftlichen Ordnung. Das große soziale Elend und die Hochflut von Reformschriften zwischen 1846 und 1849 hat E.H.Ackerknecht (1932) ${ }^{64}$ eindrucksvoll geschildert. Hier geht die politische Unruhe ebenso durch die Literatur wie durch die Medizin jener Jahre, und damit geht das Biedermeier seinem Ende entgegen.

\section{Der Sieg des Naturalismus über das Biedermeier in der Medizin}

Die Medizin der Jahre 1830 bis 1850 war, wie gesagt, der Spekulation gänzlich abhold, tatsachenhungrig, an Regeln zur Praxis interessiert. Es gab damals eine solide empirische Medizin, ja sogar mitunter eine sehr hochstehende wie bei J.L.Schönlein. Die empirischen Gesetze sollten das Pendant zu den Gesetzen der Naturwissenschaften abgeben. Der Einzug der experimentellen Idee, mit klarer Vorstellung von der Art und Weise, wie man in Physiologie und Medizin experimentieren muß, um zu mehr als empirischen, nämlich zu kausalen Gesetzen zu kommen, das vollzieht sich erst nach 1850. Die Anfänge sind bei Lotze (1843), bei Wunderlich (1842), bei Henle (1844), bei Virchow (1847) deutlich, aber die empirischen Eierschalen sind auch hier noch spürbar. E. du Bois-Reymonds Arbeiten über Elektrophysiologie (1848 ff), von Helmholtz über die Erhaltung der Kraft (1847) und über die Leitungsgeschwindigkeit im Nerven (1850) sind Signale des neuen Kausaldenkens und des endgültigen Abschieds von der Lebenskraft.

62 Fr. Oesterlen, Handbuch der medizinischen Statistik, Tübingen 1865, S. IX.

63 Ebenda, S. 9.

64 E.H.Ackerknecht, Beiträge zur Geschichte der Medizinalreform von 1848, Sudhoffs Arch. 25 (1932) 61-109, 113-183. 
Der Wortführer der neuen Richtung, CaRL Ludwig, formuliert 1852 in seinem Lehrbuch der Physiologie wohl erstmals klar die Art und Weise, wie man die Lebensvorgänge kausalanalytisch aufklären muß ${ }^{65}$ :

«1) Man bestrebt sich, den thierischen Körper in seine Bestandtheile zu zerlegen, und sucht diese letzteren, abgesehen von ihren Leistungen, innerhalb des thierischen Organismus durch möglichst scharfe Kennzeichen irgendwelcher Art von allen anderen zu scheiden. -

2) Man bestrebt sich, die von mehr oder weniger complicirten Apparaten ausgehenden Leistungen ihrem absoluten Werth nach zu messen, ohne Rücksicht auf die Art und Weise, wie diese Resultirende sich aus dem ihr zu Grunde liegenden Prozesse erzeugt. -

3) Man bestrebt sich, irgend eine Leistung als eine Funktion der sie erzeugenden Bedingungen aufzufassen; diese Aufgabe ist als die höchste der physiologischen Forschungen anzusehen. Ganz allgemein kann man sich zweier Wege bedienen, um den durch sie gebotenen Anforderungen zu genügen. a) Entweder man combinirt theoretisch (durch den mathematisch-physikalischen Calkül) oder praktisch (durch den physikalisch-chemischen Versuch) eine gewisse Summe von Bedingungen bekannter und den organischen angenäherten Eigenthümlichkeiten und vergleicht die durch sie hervorgebrachten Wirkungen mit den in der Natur erzeugten. Diese direkte Methode ist diejenige, welche sogleich zu den größten Aufschlüssen führt; aber sie ist nur selten anwendbar, sie ist aber schon mit Erfolg in Anwendung gebracht worden, z. B. als künstliche Verdauung, als Stromlauf in elastischen Röhren, als besondere electrische Combination usw., zur Aufhellung des Verdauungsprozesses, des Blutlaufes, der Muskelwirkungen usw. b) Wenn dieser Weg nicht anwendbar ist, führt ein anderer, meist nicht minder schwieriger zum Ziele; er läuft darauf hinaus, die an irgendwelchem Prozesse sich betheiligenden Bedingungen, gleichgültig ob sie sämmtlich bekannt oder nicht bekannt sind, in Gruppen zu spalten, von denen die einen constand erhalten, die anderen in meßbarer Weise verändert werde zu Zeiten, in denen man die Werthe der aus dem Prozeß hervorgehenden Leistungen mißt. Diese allgemeine Methode gibt unter den gemachten Voraussetzungen Aufschluß über den Antheil, den eine (die variable) Bedingung an der Erzielung der Gesammtleisung hat, ohne daß sie aber, wie die vorher erwähnte uns zugleich belehrte, durch welche eigenthümliche Wirkung auf die anderen Bedingungen die variable dieses Gesammtresultat erzeugen hilft.»

In diesen Ausführungen steckt in aller Knappheit das Programm der mit 1850 anhebenden neuen Zeit.

Die gleiche Entwicklung fand in jenen Jahren in Frankreich statt. Claude Bernard (1813-1878) ${ }^{66}$ betonte schon in seiner Antrittsvorlesung 1855 am Collège de France, daß die Beobachtungsmedizin nicht weiter führe. Die zukünftige Medizin müsse von der experimentellen Methode

${ }^{65}$ Carl Ludwig, Lehrbuch der Physiologie des Menschen, Leipzig/Heidelberg, 1. Auflage, I. Band 1852, II. Band 1856, Zitat aus Band I, S.11,12,13, siehe auch RoтнsснUн, Anm. 24.

${ }^{66} \mathrm{Zu}$ Claude Bernard erschien kürzlich: Philosophie et Méthodologie scientifiques de Claude Bernard (Coll. Internat., Strasbourg 1965), Paris 1967, mit Beiträgen vieler Autoren und charakteristischen Zitaten Bernards zur experimentellen Medizin. 
durchsetzt werden. Das wird in den nächsten Jahren von ihm immer klarer ausgesprochen und hat schließlich auch praktisch zu großen Erfolgen geführt. Die Introduction à l'étude de la médecine expérimentale 1865 ist die Apotheose dieser Richtung geworden ${ }^{67}$. Das ist yon den Medizinhistorikern immer wieder dargelegt worden. In seinen Principes de médecine expérimentale (etwa 1872, erst 1947 publiziert) heißt es: «L'idée de la médecine expérimentale est l'idée de toutes les sciences expérimentales. Dominer scientifiquement la nature vivante, la conquérir au profit de l'homme: telle est l'idée fondamentale du médecin expérimentateur. ${ }^{68}$

Ein alter Arzt jener Zeit, Dr. Volz, bemerkt am 16. September 1858 auf der 34. Versammlung. Deutscher Naturforscher und Ärzte in Carlsruhe, daß seine Generation viel medizinische Geschichte und eine Wandlung erlebt habe, wie sie $2000 \mathrm{Jahre} \mathrm{nicht} \mathrm{zustande} \mathrm{gebracht} \mathrm{haben.} \mathrm{Diese} \mathrm{Jahre} \mathrm{waren}$ schwer. Jetzt liegt die Zukunft offen, der Weg ist erkannt. Es ist der Weg, der auf Gesetze baut. "Unsere medizinische Forschung ist noch die der Thatsachen, die unserer Söhne soll die der Ursache, der Gesetze sein. ${ }^{69}$ Dieser Weg ging von der naturphilosophischen Medizin über die empirische, vorexperimentelle, skeptische Medizin des Biedermeiers, die die Morgenröte der Zukunft ahnt, ohne den Weg dahin klar zu erkennen, in die Zeit der naturalistischen Medizin nach der Jahrhunderthälfte. Ihre Vorläufer waren in Deutschland u.a. Schwann, Lotze, Wunderlich, Henle, Virchow und Oesterlen. Sie alle suchten, ihrer ungenügenden Gegenwart durch kühne Vorgriffe auf die Zukunft ein neues Ziel zu geben.

\section{Zusammenfassung}

Die romantisch-idealistische bzw. naturphilosophische Epoche der deutschen Kultur und Medizin schwächt sich seit 1815 merklich ab und klingt um 1830 aus. Um 1850 aber beginnt nach einigen Vorwehen in den vierziger Jahren eine kräftige realistisch-naturalistisch-materialistische Strömung. Die Jahrzehnte zwischen 1830 und 1850 stellen in Zeitgeist, Mode, Literatur und Kunst den Höhepunkt des Biedermeiers dar. Ihnen entspricht eine Medizin, die ebenfalls gewisse einheitliche und zum Biedermeier passende Züge erkennen läßt. Man ist in diesen beiden Jahrzehnten beseelt von Eifer,

67 Introduction in deutscher Übersetzung (vgl. Anm.43).

68 Principes de médecine expérimentale, publ. par Léon Delhoume, Paris 1847, S. 89.

69 Dr. Vouz, Ansprache vor der 34. Versammlung Deutscher Naturforscher und Ärzte, Carlsruhe, September 1858, S. 13,14. 
neue Erfahrungen zu machen. Man ist geradezu erfahrungssüchtig, es wird gesammelt, beschrieben, benannt, verglichen, klassifiziert. Man hält sich in der Generalisierung betont zurück, das Einzelne verdient eher das liebevolle Interesse als das Allgemeine. Man scheut die Spekulation und liebt die Beobachtung, man beharrt auf dem Vordergründigen. Anatomie, Physiologie und Klinik bevorzugen die deskriptive Methode der Vergleichung, der erfahrungsgemäßen Korrelation der Erscheinungen und Umstände. Man sucht nach empirischen Gesetzen, die möglichst numerisch gesichert sein sollen. Die Gabe, die Natur durch den Versuch planmäßig zu befragen, ist noch wenig entwickelt. Das Zeitalter ist trotz seiner Versuche noch fast «vorexperimentell» zu nennen. Seine Gesamthaltung ist betont empirisch. Durch die Ärztewelt zieht der Geist äußerster Ernüchterung, man ist theorienfeindlich und von großer therapeutischer Skepsis erfüllt. Die bahnbrechenden Geister jener Jahre ahnen zwei Auswege aus dem Dilemma der Medizin, erstens die Einführung des naturwissenschaftlichen, methodischen, kausalanalytischen Experiments in die Forschung und zweitens die Entwicklung einer prophylaktischen Medizin und sozialen Hygiene in der Praxis. Es sei vorgeschlagen, diese so charakterisierte Epoche von 1830 bis 1850 als «Biedermeiermedizin» zu bezeichnen. Gegen Ende dieser Zeit bricht überall die Unruhe aus, politische Unruhe, Unruhe im ärztlichen Beruf, Ruf nach Medizinalreform, Entrüstung über die soziale Lage der Arbeiterklasse, Forderung nach einer Reform der Gesellschaft und des Gesundheitswesens usw. Damit überschreitet die Entwicklung jene Jahrhunderthälfte, die in der Tat eine erhebliche Zäsur in der medizinischen Kulturgeschichte darstellt. 